\title{
Modelos matemáticos para estimar o consumo de dieta para Tilápia do Nilo (Oreochromis niloticus) e Tambaqui (Colossoma macropomum)
}

Mathematical models to estimate diet intake for Nile Tilapia (Oreochromis niloticus) and Tambaqui (Colossoma macropomum)

Modelos matemáticos para estimar la ingesta dietética de Tilapia del Nilo (Oreochromis niloticus) y

Tambaqui (Colossoma macropomum)

\author{
Antonio Wilson Fornero Aguiar \\ (ORCID: https://orcid.org/0000-0001-7386-7789 \\ Universidade Federal Rural do Rio de Janeiro, Brasil \\ Fundação Instituto de Pesca do Estado do Rio de Janeiro, Brasil \\ E-mail: aguirawf@gmail.com \\ Matheus Pereira dos Santos \\ ORCID: https://orcid.org/0000-0001-6341-1569 \\ Universidade Federal Rural do Rio de Janeiro, Brasil \\ E-mail: matheusps.pereira@gmail.com \\ Giselle Eler Amorim Dias \\ ORCID: https://orcid.org/0000-0002-4066-3824 \\ Fundação Instituto de Pesca do Estado do Rio de Janeiro, Brasil \\ E-mail: giselleeler@gmail.com \\ Marcelo Maia Pereira \\ ORCID: https://orcid.org/0000-0002-1898-2722 \\ Fundação Instituto de Pesca do Estado do Rio de Janeiro, Brasil \\ E-mail: mmaiap2001@yahoo.com.br
}

\begin{abstract}
Resumo
A tecnologia de precisão a cada dia se insere ao meio rural, e para isso, pesquisas com modelos matemáticos que possam ser adotadas para aperfeiçoar os manejos se fazem necessárias. Essa pesquisa tem o objetivo de elaborar curvas não lineares a partir de equações consolidadas no meio científico para o consumo de dietas para tambaqui e Tilápia do Nilo. Ao obter informações sobre o crescimento ao longo do tempo e tabelas de oferta de alimentos para cada fase de crescimento para a espécie de peixes estudadas, se procedeu à elaboração de curvas de crescimento ao longo do tempo e de seu consumo alimentar. Com base nos dados coletados, os parâmetros das equações de quatro modelos não lineares (Gompertz e Logístico) foram estimados para as duas variáveis estudadas (Crescimento em peso (g) ao longo do tempo e Consumo alimentar (g) ao longo do tempo). Avaliadores de ajustes (interpretação biológica e análise dos resíduos studentizados) foram adotados para selecionar e verificar a qualidade dos modelos. Os dados gerados de oferta ou consumo de ração para tilápias do Nilo e tambaquis se adequaram aos modelos Gompertz e Logístico, respectivamente. Esses modelos podem ser utilizados para estimar consumo ou oferta de ração para tilápia do Nilo e o tambaqui, porém é necessário rigor nos critérios de avaliação e ajustes.
\end{abstract}

Palavras-chave: Alimentos; Curvas; Equações; Crescimento.

\begin{abstract}
Precision technology is inserted into rural areas every day, and for that, research with mathematical models that can be adopted to improve management is necessary. This research aims to develop non-linear curves from equations consolidated in the scientific world for the consumption of diets for tambaqui and Nile tilapia. By obtaining information on growth over time and food supply tables for each growth phase for the studied fish species, we proceeded to draw up growth curves over time and their food consumption. Based on the data collected, the parameters of the equations of four nonlinear models (Gompertz and Logistic) were estimated for the two studied variables (Growth in weight (g) over time and Food consumption (g) over time. over time). Adjustment evaluators (biological interpretation and analysis of studentized residues) were adopted to select and verify the quality of the models. The data generated on the supply or consumption of feed for Nile tilapia and tambaqui fit the Gompertz and Logistic models, respectively. These models can be used to estimate feed consumption or supply for Nile tilapia and tambaqui, but strict evaluation and adjustment criteria are required.
\end{abstract}

Keywords: Food; Curves; Equations; Growth. 


\section{Resumem}

La tecnología de precisión se inserta en las áreas rurales todos los días, y para eso es necesaria la investigación con modelos matemáticos que se puedan adoptar para mejorar la gestión. Esta investigación tiene como objetivo desarrollar curvas no lineales a partir de ecuaciones consolidadas en el mundo científico para el consumo de dietas para tambaqui y tilapia del Nilo. Al obtener información sobre el crecimiento en el tiempo y tablas de suministro de alimentos para cada fase de crecimiento de las especies de peces estudiadas, se procedió a la elaboración de curvas de crecimiento en el tiempo y su consumo de alimentos. Con base en los datos recolectados, se estimaron los parámetros de las ecuaciones de cuatro modelos no lineales (Gompertz y Logistic) para las dos variables estudiadas (Crecimiento en peso ( $\mathrm{g}$ ) a lo largo del tiempo y Consumo de alimentos ( $\mathrm{g}$ ) a lo largo del tiempo. tiempo extraordinario). Se adoptaron evaluadores de ajuste (interpretación biológica y análisis de residuos studentizados) para seleccionar y verificar la calidad de los modelos. Los datos generados sobre el suministro o consumo de alimento para la tilapia del Nilo y el tambaqui se ajustan a los modelos de Gompertz y Logístico, respectivamente. Estos modelos pueden usarse para estimar el consumo o suministro de alimento para la tilapia del Nilo y el tambaqui, pero se requieren criterios estrictos de evaluación y ajuste.

Palabras clave: Alimentos; Curvas; Ecuaciones; Crecimiento.

\section{Introdução}

A aplicabilidade do manejo alimentar embasado em modelos matemáticos de consumo de ração é importante nos processos produtivos, que tendem a melhorar tanto os aspectos econômicos, quanto os aspectos técnicos nas mais diversas atividades. Na piscicultura, o manejo alimentar integrado a um banco de dados quantitativos, pode fornecer a dosagem ideal de alimentos para cada indivíduo em cada fase de desenvolvimento de acordo com fatores inerentes à qualidade da água. Dentre os principais fatores, cita-se a temperatura e oxigênio dissolvido (Dumas et al., 2010).

Alterações metabólicas e fisiológicas ocasionadas pelo aumento ou redução da temperatura do ambiente aquático são determinantes para organismos ectotérmicos em sistema produtivo, principalmente em relação ao consumo de alimentos (Cyrino et al., 2010).

Nos peixes, quando fora da faixa ideal do conforto térmico, a quantidade de ração oferecida pode ser inadequada (Workagegn, 2012). Para além do fato dos peixes apresentarem aproveitamento restrito do nitrogênio e fósforo para o crescimento, nas dosagens incorretas, a ração é consumida parcialmente, ocasionando perdas para o meio, e consequentemente deterioração da água, que contará com maior quantidade de material orgânico em decomposição (Boyd, 2000; Lazur, 2007).

Diretamente relacionado à temperatura e salinidade, o oxigênio dissolvido na água representa o principal fator químico para o bom desempenho metabólico de organismos aquáticos com respiração branquial (Tsadik \& Kutty, 1987; Kim, 2017).

Com o emprego da aquicultura intensiva, com densidades de estocagem superiores, a manutenção de lotes homogêneos se torna uma grande limitação para a criação comercial menos segmentada. A competição por alimento, principalmente na fase inicial da criação, representa principal fator para a diferença no consumo e ganho de peso dos animais (El-Sayed, 2002).

O manejo de classificação torna-se necessário quando há grandes diferenças de peso entre os animais do mesmo lote. A prática da classificação é negativa do ponto de vista do bem estar animal, uma vez que o manejo é mecânico, podendo causar queda de escamas, ferimentos que propiciam infecções bacterianas, desafios ao sistema imunológico, estresse e alterações metabólicas que afetam o consumo de ração (Kubitza, 2000).

A aplicação de taxas mais precisas de consumo de ração possibilitam aplicar diferentes estratégias de arraçoamento, com frequências e quantidades diferentes, o que propicia maior homogeneidade nos lotes. As aplicações de tecnologias de precisão possibilitam o emprego de alimentadores automatizados, utilitários essenciais para a execução prática dos modelos padronizados de consumo. Para algumas espécies, existe ainda a possibilidade da alimentação noturna, como um incremento em prática viável com utilização da automação (Hossain et al., 2001; Souza et al., 2012). 
O aperfeiçoamento dos fatores inerentes à alimentação, com ajustes constantes de consumo, leva à possibilidade de melhorar os índices zootécnicos, ao proporcionar melhores resultados em relação ao ganho de peso diário, menor tempo por ciclo, menor taxa de mortalidade na fase juvenil e demais fatores econômicos e ambientais, como a economia de ração, com menor potencial poluidor por eutrofização do ambiente aquático (Pereira et al., 2014).

Ao apresentar características semelhantes de ectotermia, e com relevante respiração branquial, a tilápia (Oreochromis niloticus) e o tambaqui (Colossoma macropomum) demandam temperaturas específicas e taxas adequadas de oxigênio dissolvido na água para realizar efetiva metabolização do alimento ingerido. Para tanto, o controle das condições de criação em cativeiro são essenciais para favorecer o máximo aproveitamento da ração, de modo a gerar maior eficiência do sistema produtivo. Ao considerar os gastos com ração e acondicionamento do ambiente no sistema produtivo, a administração de tais recursos se torna de essencial importância para a competitividade do setor produtivo. (Cyrino et al., 2010).

Em condições específicas de hipóxia, comum em ambiente aquático amazônico, o tambaqui possui adaptações que otimizam a captação de oxigênio, como o aumento da taxa de ventilação branquial (Araújo-Lima \& Goulding, 1998).

Uma adaptação morfológica observada no tambaqui é a expansão do lábio inferior, quando o nível de oxigênio fica inferior a $0.5 \mathrm{mg} / \mathrm{L}$. Ao aumentar a superfície de contato com a formação de um edema, há maior facilidade na captação de oxigênio na camada superficial da água, onde a concentração de oxigênio dissolvido é maior (Val et al., 1998).

Em cativeiro, tal adaptação representa uma vantagem para o melhor aproveitamento da metabolização da ração ofertada, em momentos de baixa concentração de oxigênio. Sendo assim, a maior compreensão das demandas específicas por alimento, de acordo com parâmetros como temperatura e oxigênio dissolvido, são ferramentas importantes para a realização de ajustes mais precisos no manejo de arraçoamento das espécies de peixes.

Com o monitoramento e ajustes, a partir da leitura, registro e formatação de banco de dados em curvas de crescimento, as atividades aquícolas podem se integrar ao conceito de precisão com a modulação do crescimento animal (Chizzotti \& Valente, 2014).

A aquicultura nacional, embasada em uma expansão planejada, seguindo critérios de aptidão para a aquisição de crédito, fará da atividade aquícola ainda mais profissional, produtiva e competitiva num setor que apresenta crescentes demandas, principalmente aliada a tecnologia da precisão.

Com isso, o presente trabalho teve como objetivo verificar o comportamento do ajuste dos dados para consumo alimentar (g) das espécies tilápia do Nilo e tambaqui, aos parâmetros dos modelos de Gompertz, Von Bertalanffy, Brody e Logística, bem como determinar modelos matemáticos para estimar consumo alimentar (g) em função do tempo para tilápia do Nilo e Tambaqui.

\section{Metodologia}

Primeiramente, procedeu-se uma seleção de modelos de crescimento corporal (g) para as espécies Tilápia do Nilo (Oreochromis niloticus) e Tambaqui (Colossoma macropomum).

O modelo de crescimento corporal adotado para tilápia do Nilo foi o de Gompertz (Zardin et al., 2019), e o modelo de crescimento corporal adotado para tambaqui foi o Logístico (Mourad et al., 2018). As informações consideradas importantes na influência do consumo alimentar na criação de peixes, como rações utilizadas, temperatura de cultivo e outros fatores de importância foram identificados para interpretação dos resultados.

$\mathrm{Na}$ sequência, selecionou-se uma tabela de oferta de ração as duas espécies estudadas. Normalmente, as informações são em \% em relação ao peso vivo ou biomassa no local de criação.

`A tabela adotada para cálculo de oferta de ração para tilápia do Nilo foi adaptada por profissionais da Fundação Instituto de Pesca do Estado do Rio de Janeiro a partir de vários documentos (Tabela 1). A tabela para cálculo de oferta de 
ração para tambaqui foi adaptada pela empresa Lupus Alimentos (TOP CRIADOR Peixes®) para arraçoamento de peixes redondos (Tabela 2).

As tabelas e curvas foram construídas e elaboradas a partir dos modelos encontrados e selecionados de crescimento corporal (g) e de tabelas de oferta de ração para cada espécie em estudo. Posteriormente, foram feitos cálculos da ração a ser ofertada a partir do modelo de crescimento dos peixes adotando as tabelas de alimentação selecionadas. Dois cálculos foram realizados: um de oferta diária e outra acumulativa. Todos os cálculos foram realizados através do Microsoft Office Excel (2007).

Esses dados foram utilizados para estimar os parâmetros de dois modelos não lineares (Gompertz para tilápia do Nilo e Logístico para tambaqui), devido a manter a qualidade estatística, foi repetido para estimar o consumo ou oferta de ração, no mesmo modelo do crescimento corporal dos peixes.

Tabela 1. Recomendação de taxa de arraçoamento (\%) para tilápia do Nilo (Oreochromis niloticus) de acordo com peso médio (g) dos peixes e temperatura da água de criação*.

\begin{tabular}{ccc}
\hline Peso médio $(\mathrm{g})$ & Taxa de alimentação \% & Temperatura ${ }^{\circ} \mathrm{C}$ \\
\hline$(1-5)$ & 12,00 & $24-26$ \\
$(5-10)$ & 6,40 & $24-26$ \\
$(10-20)$ & 5,60 & $24-26$ \\
$(20-50)$ & 4,00 & $24-26$ \\
$(50-70)$ & 3,20 & $24-26$ \\
$(70-100)$ & 3,20 & $24-26$ \\
$(100-150)$ & 2,40 & $24-26$ \\
$(150-200)$ & 2,16 & $24-26$ \\
$(200-300)$ & 1,92 & $24-26$ \\
$(300-400)$ & 1,60 & $24-26$ \\
$(400-500)$ & 1,50 & $24-26$ \\
$(500-550)$ & 1,40 & $24-26$ \\
$(550-700)$ & 1,40 & $24-26$ \\
$(700-1000)$ & 1,00 & $24-26$ \\
\hline
\end{tabular}

Fonte: *Adaptada da Fundação Instituto de Pesca do estado do Rio de Janeiro.Ano?

Tabela 2. Recomendação de taxa de arraçoamento (\%) para tambaqui (Colossoma macropomum) de acordo com peso médio (g) dos peixes e temperatura da água de criação*.

\begin{tabular}{ccc}
\hline Peso Médio $(\mathrm{g})$ & Taxa de alimentação $\%$ & Temperatura ${ }^{\circ} \mathrm{C}$ \\
\hline $0,5-2,0$ & 18,0 & 26 a 30 \\
$2,0-15,0$ & 12,0 & 26 a 30 \\
$15,0-200,0$ & 9,0 & 26 a 30 \\
$200,0-400,0$ & 6,0 & 26 a 30 \\
$400,0-800,0$ & 3,5 & 26 a 30 \\
$800,0-1.000,0$ & 2,5 & 26 a 30 \\
$>1.000,0$ & 1,0 & 26 a 30 \\
\hline
\end{tabular}

Fonte:*Adaptada pela empresa Lupus Alimentos (TOP CRIADOR Peixes®) para arraçoamento de peixes redondos. 


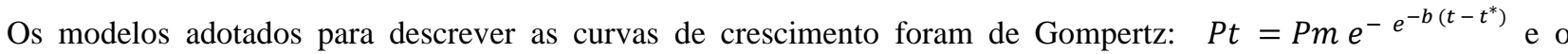
Logístico: $P t=P m /\left(1+B e^{(-k t)}\right)$, em que $P t=$ peso $(\mathrm{g})$ ao tempo $\mathrm{t}$, estimado em função do $\mathrm{Pm} ; \mathrm{Pm}=\mathrm{peso}(\mathrm{g})$ à maturidade; $\mathrm{b}=$ taxa de maturidade $(\mathrm{g} / \mathrm{dia}) ; \mathrm{B}=$ valor sem interpretação biológica; $\mathrm{t}^{*}=$ tempo (dias) em que a taxa de crescimento é máxima; $\mathrm{t}$ = tempo (dias); $\mathrm{k}$ = taxa relativa de crescimento.

As equações utilizadas para descrever a curva de consumo da dieta foram a de Gompertz, $C t=C m e^{-e^{-b\left(t-t^{*}\right)}}$ e a Logística, $\left.C t=C m /\left(1+B e^{(-k t}\right)\right)$ em que $C t=$ consumo da dieta $(\mathrm{g})$ pelo animal ao tempo $\mathrm{t}$, expresso em função do $\mathrm{Cm} ; \mathrm{Cm}=$ consumo da dieta $(\mathrm{g})$ à maturidade do animal; $\mathrm{b}=$ taxa de consumo $(\mathrm{g} / \mathrm{dia}) ; \mathrm{t}^{*}=$ tempo (dias) em que a taxa de consumo é máxima; $\mathrm{t}$ = tempo (dias) $\mathrm{B}=$ valor sem interpretação biológica; $\mathrm{k}=$ taxa relativa de crescimento.

Para estimar os parâmetros das equações matemáticas, foi utilizado o procedimento NLIN do Sistema de Análises Estatísticas, SAS (2001). As estimativas dos parâmetros foram obtidas por método iterativo de Gauss-Newton, método modificado, desenvolvido por Hartley (1961), para modelos não-lineares.

Avaliadores de ajustes foram adotados para verificar a qualidade dos modelos e os critérios utilizados foram: coeficiente de determinação $\left(\mathrm{R}^{2}\right)$ (Souza, 1998); interpretação biológica e a distribuição dos resíduos studentizados, usada para detectar pontos discrepantes, em geral, considera-se uma observação marginalmente discrepante se $\mid$ Rti* $\mid>3$ (Lemonte, 2008).

\section{Resultados e Discussão}

Os valores estimados para crescimento corporal para tilápia do Nilo (Zardin et al., 2019) e consumo ou oferta acumulada de ração para modelo de Gompertz se mostraram adequados quanto a interpretação biológica dos valores (Tabela 3). $O R^{2}$ foi de 0,9622 para a estimativa do consumo ou oferta de ração para tilápia do Nilo.

Tabela 3. Valores estimados para as equações de Gompertz para crescimento da tilápia do Nilo (Oreochromis niloticus) e oferta de ração.

\begin{tabular}{cccc}
\hline Parâmetro & Pm ou Cm $(\mathrm{g})$ & $\mathrm{b}$ & $\mathrm{t}^{*}$ \\
\hline Crescimento $^{*}$ & $1.100,00$ & 0,0068 & 308,0 \\
Consumo ou oferta de & $4.353,80$ & 0,0047 & 425,1 \\
ração & & & \\
\hline
\end{tabular}

$\mathrm{Pm}=$ peso $(\mathrm{g})$ à maturidade; $\mathrm{b}=$ taxa de maturidade $(\mathrm{g} / \mathrm{dia}) ; \mathrm{t}^{*}=$ tempo (dias) em que a taxa de crescimento é máxima; $\mathrm{t}=$ tempo (dias); $\mathrm{Cm}=$ consumo da dieta $(\mathrm{g})$ à maturidade do animal. *Valores estimados por Zardin et al., 2019.

A partir da curva de crescimento para tilápia do Nilo através do modelo de Gompertz foi calculada a oferta de ração diária e com isso uma curva com valores acumulados foi obtida e depois esses valores foram base para estimar o consumo ou oferta de ração através do modelo de Gompertz (Figura 1). 
Figura 1. Curva para crescimento de tilápia do Nilo (Oreochromis niloticus) estimada pelo modelo de Gompertz (Zardin et al., 2019), curva de oferta de ração para tilápia do Nilo (calculada) e curva de oferta de ração para tilápia do Nilo estimada pelo modelo de Gompertz.

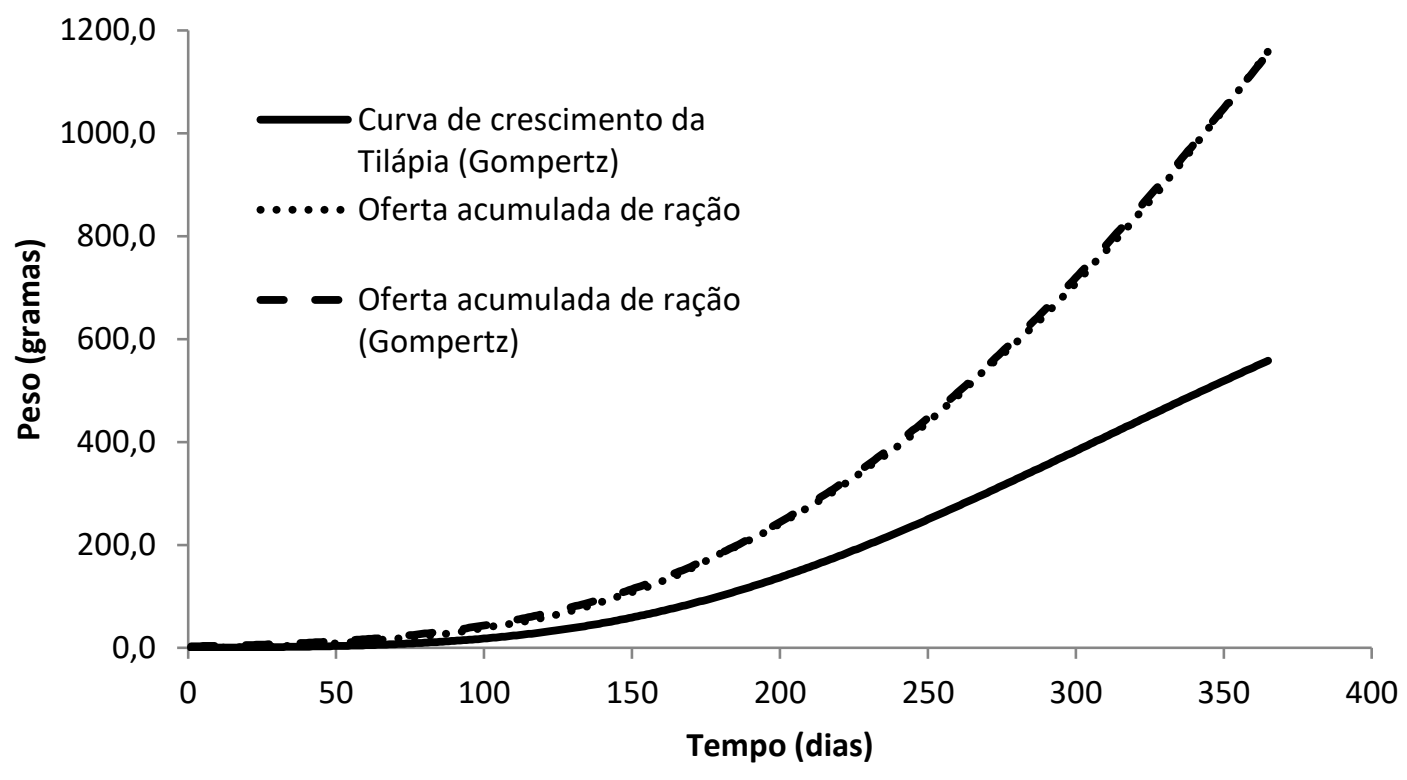

Fonte: Autores.

Uma ferramenta para verificar adequação de um modelo para consumo ou oferta de ração é verificar estimativa de conversão alimentar. Estimativa de conversão alimentar para tilápia do Nilo a partir do valores estimados para crescimento (g) pelo modelo de Gompertz e dos valores calculados para oferta de ração, apresentaram valores acima de 2 g/g no inicio e no final do crescimento dos animais (Figura 2) e os valores se agruparam como se fossem fases do crescimento.

Figura 2. Estimativa de conversão alimentar para tilápia do Nilo (Oreochromis niloticius) a partir de valores estimados para crescimento (g) pelo modelo de Gompertz e dos valores calculados para oferta de ração.

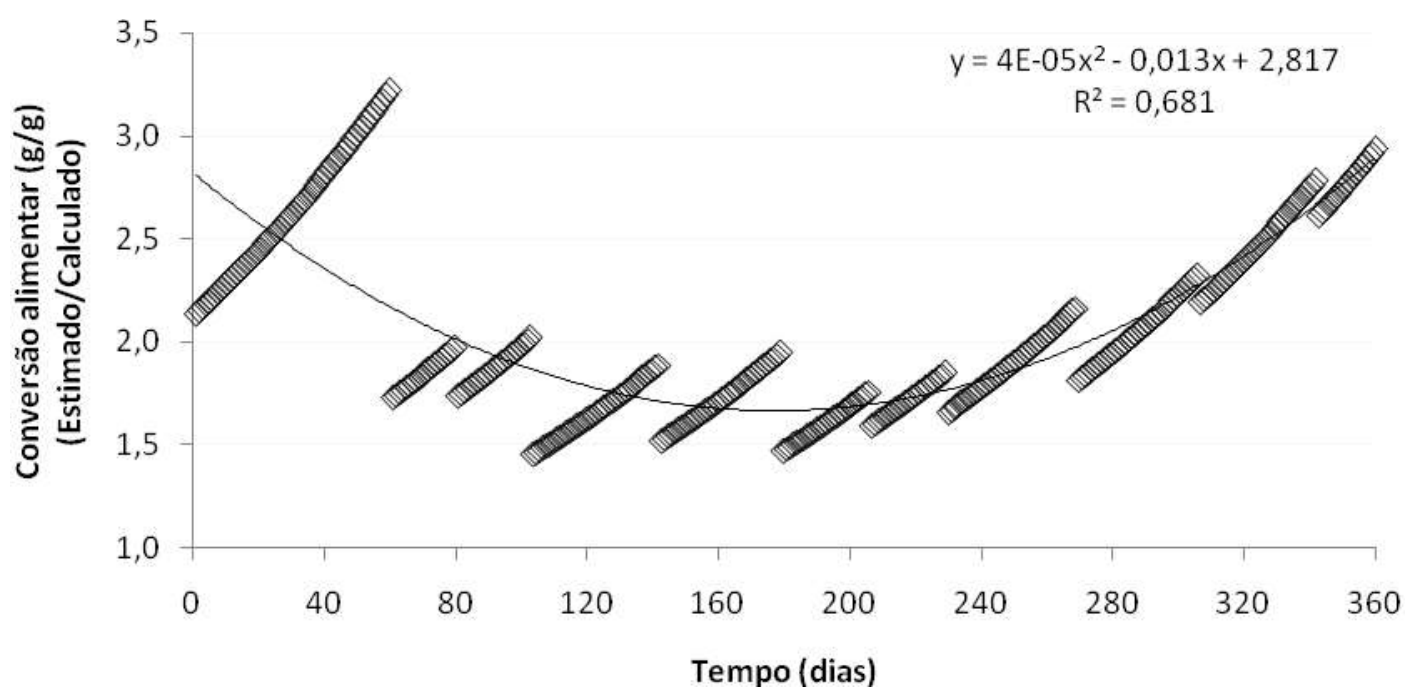

Fonte: Autores. 
A estimativa de conversão alimentar para tilápia do Nilo a partir dos valores estimados para crescimento (g) pelo modelo de Gompertz e dos valores também estimados para oferta de ração, apresentaram valores acima de $2 \mathrm{~g} / \mathrm{g}$ no inicio e no final do crescimento dos animais (Figura 3), porém os valores de conversão alimentar se apresentaram sequenciais. A conversão alimentar da tilápia do Nilo varia conforme a fase de criação, mas segue de 1,1 até 2,0 g/g em geral (Logato, 2000).

Figura 3. Estimativa de conversão alimentar para tilápia do Nilo (Oreochromis niloticus) a partir de valores estimados para crescimento (g) pelo modelo de Gompertz e dos valores estimados pelo modelo de Gompertz para oferta de ração.

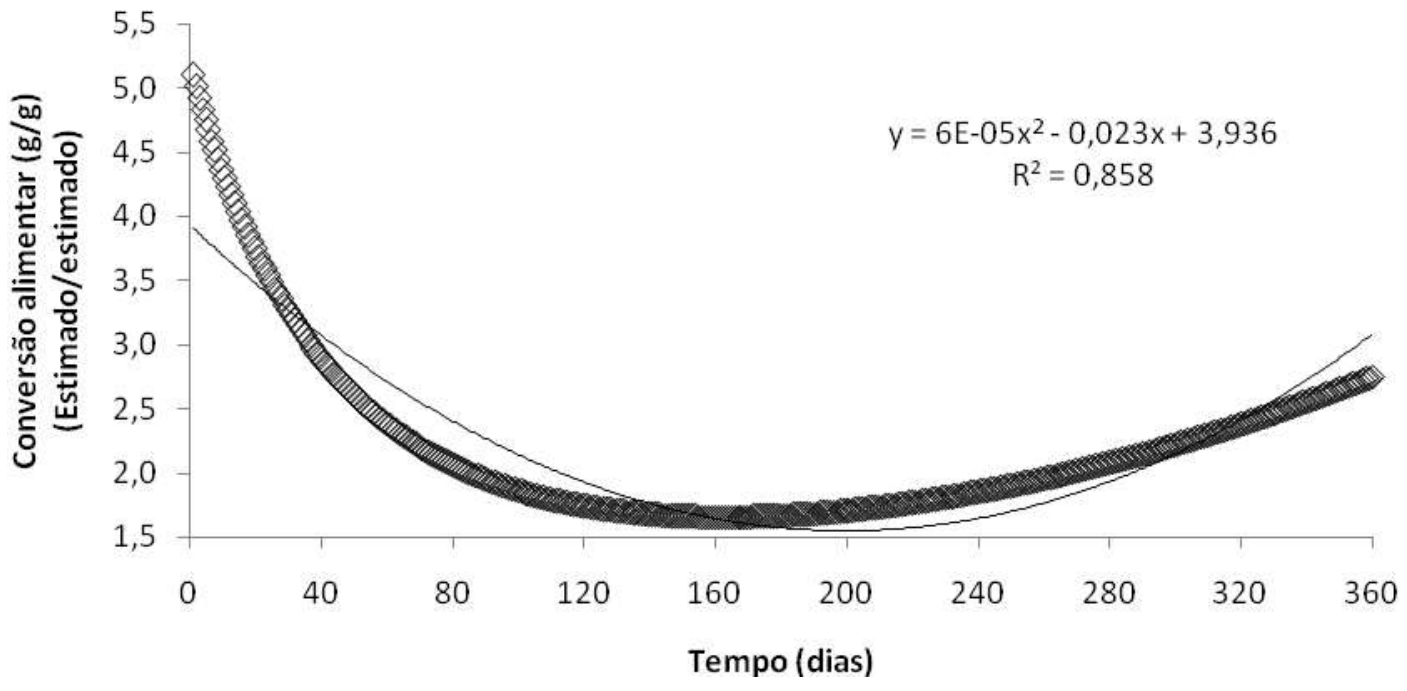

Fonte: Autores.

Os valores dos resíduos Studentizados confimam que há uma possibilidade do uso deste recurso para estimar a oferta e o consumo alimentar das tilápias do Nilo, porém os valores foram superestimados e apresentaram outliers na parte final das curvas (Figura 4).

Figura 4. Valores de resíduos studentizados calculados para estimativas do modelo de Gompertz para oferta de ração para tilápia do Nilo (Oreochromis niloticus).

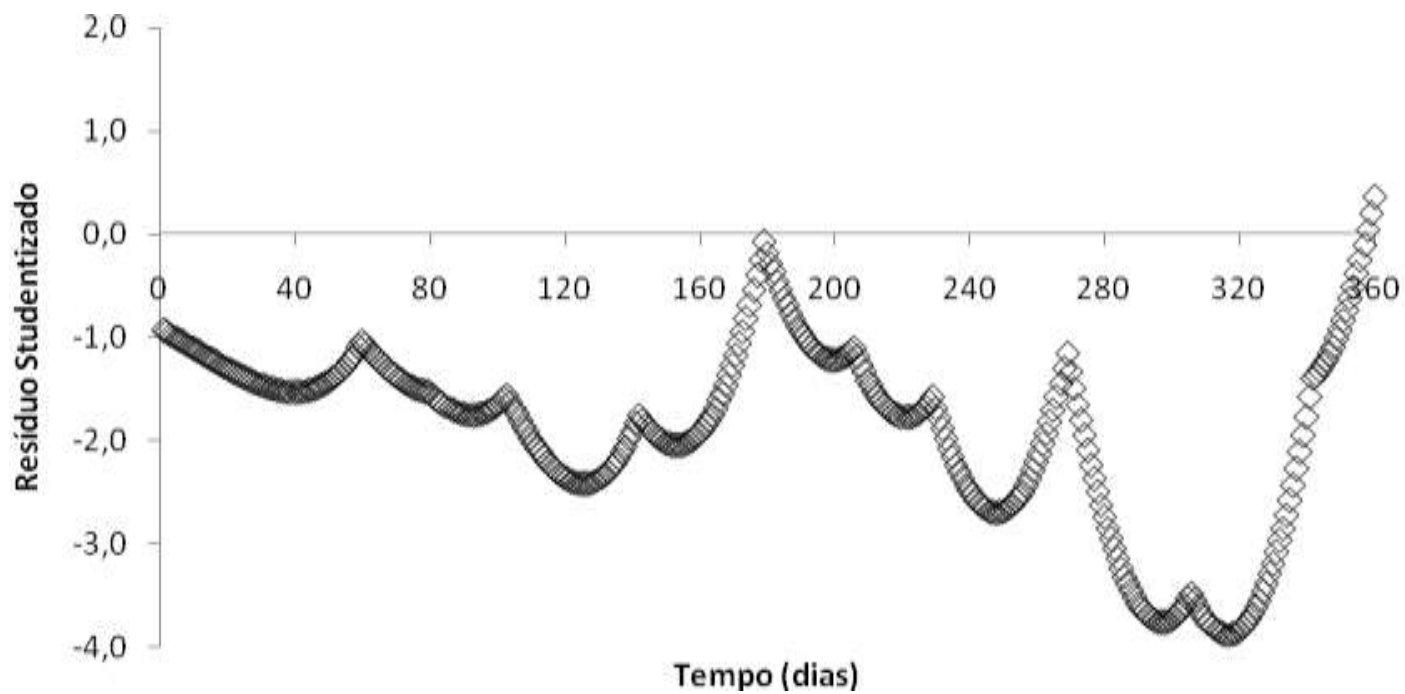

Fonte: Autores. 
Os valores estimados para crescimento corporal para tambaqui (Mourad et al., 2018) e consumo ou oferta acumulada de ração para modelo de Logístico se mostraram adequados quanto a interpretação biológica dos valores (Tabela 4). O $\mathrm{R}^{2}$ foi de 0,9845 para a estimativa do consumo ou oferta de ração para tambaqui.

Tabela 4. Valores estimados para as equações do modelo Logístico para crescimento de tambaqui (Colossoma macropomum) e oferta de ração.

\begin{tabular}{cccc}
\hline Parâmetro & Pm ou Cm & B & $\mathrm{k}$ \\
\hline Crescimento $(\mathrm{g})^{*}$ & $1.056,0$ & 29,57 & 0,0343 \\
Consumo ou oferta de & $4.339,9$ & 35,24 & 0,0205 \\
ração $(\mathrm{g})$ & & & \\
\hline
\end{tabular}

$\mathrm{Pm}=$ peso $(\mathrm{g})$ à maturidade; $\mathrm{B}=$ valor sem interpretação biológica; $\mathrm{k}=$ taxa relativa de crescimento. $\mathrm{Cm}=$ consumo da dieta (g) à maturidade do animal. * Fonte: valores estimados por Mourad et al. (2018).

A partir da curva de crescimento para tambaqui através do modelo Logístico foi calculada a oferta de ração diária e com isso uma curva com valores acumulados foi obtida e posteriormente, esses valores representaram base para estimar o consumo ou oferta de ração através do modelo Logístico (Figura 5). Os valores calculados para consumo ou oferta de ração se assemelham com o estimado pelo modelo Logístico.

Figura 5. Curva para crescimento de tambaqui (Colossoma macropomum) estimada pelo modelo Logístico (Mourad et al., 2018), curva de oferta de ração para tambaqui (calculada) e curva de oferta de ração para tambaqui estimada pelo modelo Logístico.

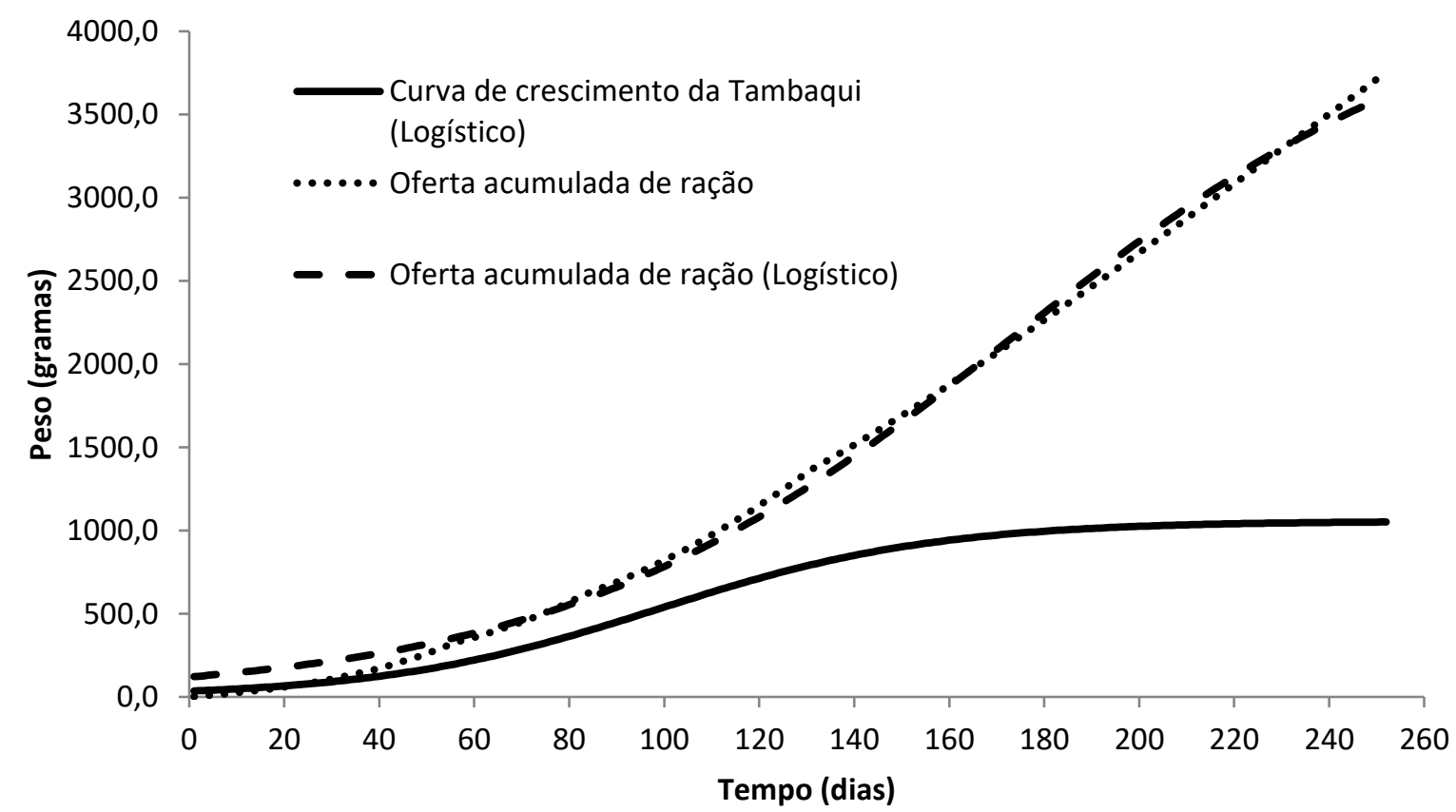

Fonte: Autores.

A conversão alimentar estimada apresentou valores muito discrepantes e com isso foi necessário apresentar dois cenários, sendo até 260 dias de criação do tambaqui (Figura 6 e 8) e até 180 dias (Figura 7 e 9). A conversão alimentar do tambaqui variou de 1,5 a 2,5 g/g de acordo com a fase de crescimento (Logato, 2000). 
Figura 6. Estimativa de conversão alimentar para tambaqui (Colossoma macropomum) a partir dos valores estimados para crescimento (g) pelo modelo de Logístico e dos valores calculados para oferta de ração até 252 dias.

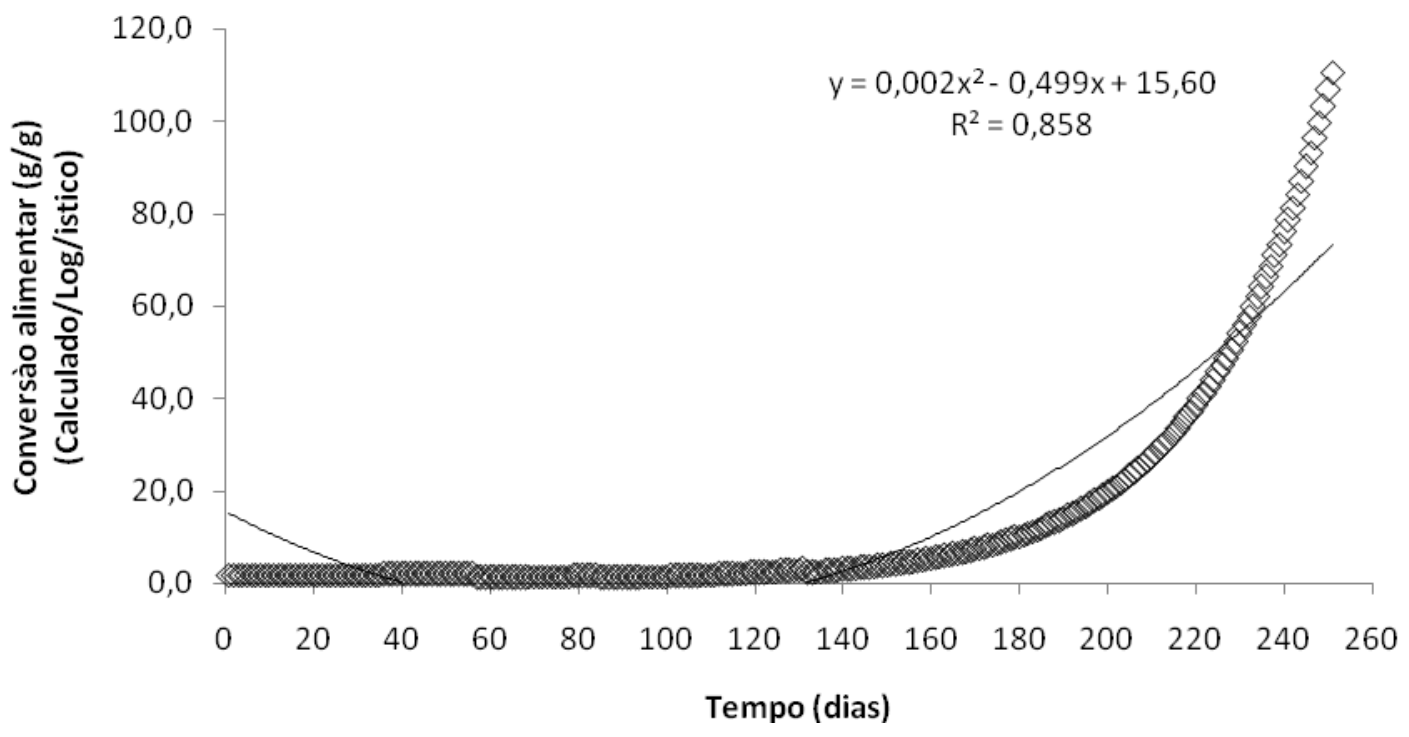

Fonte: Autores.

Figura 7. Estimativa de conversão alimentar para tambaqui (Colossoma macropomum) a partir do valores estimados para crescimento (g) pelo modelo de Logístico e dos valores calculados para oferta de ração até 180 dias.

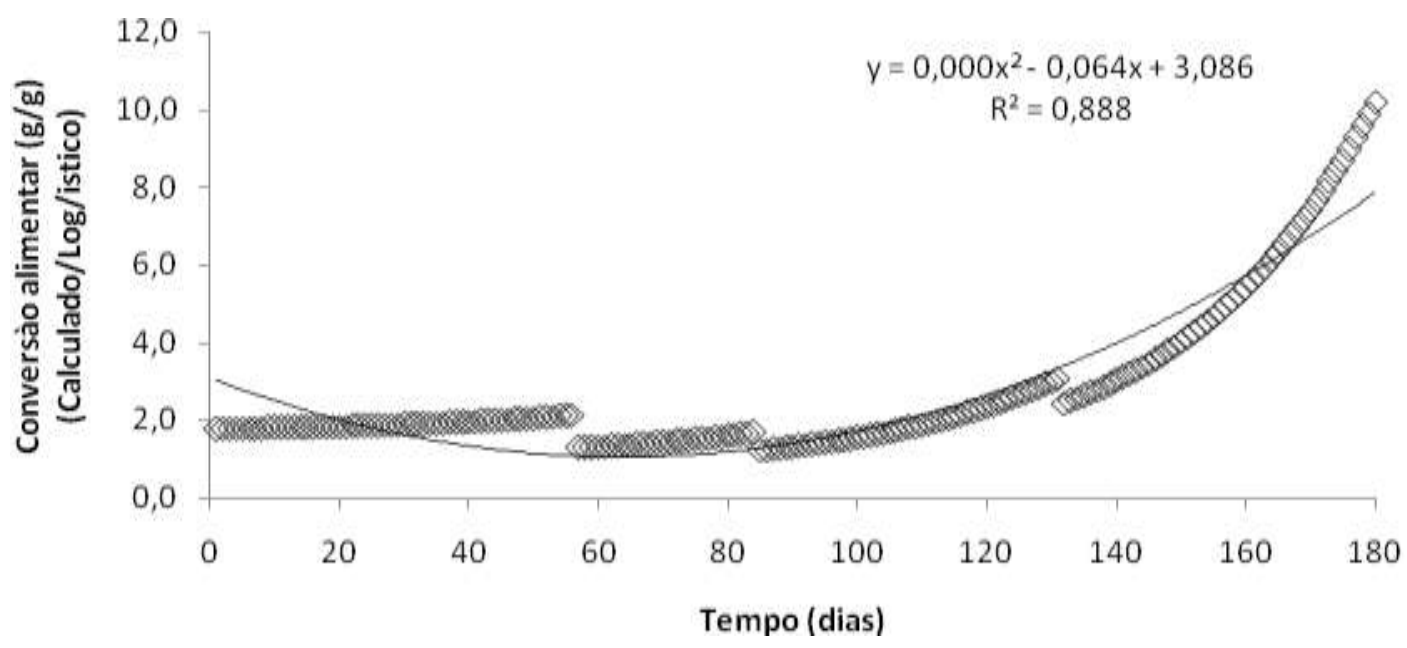

Fonte: Autores. 
Figura 8. Estimativa de conversão alimentar para tambaqui (Colossoma macropomum) a partir de valores estimados para crescimento (g) pelo modelo Logístico e dos valores estimados pelo modelo Logístico para oferta de ração até 252 dias.

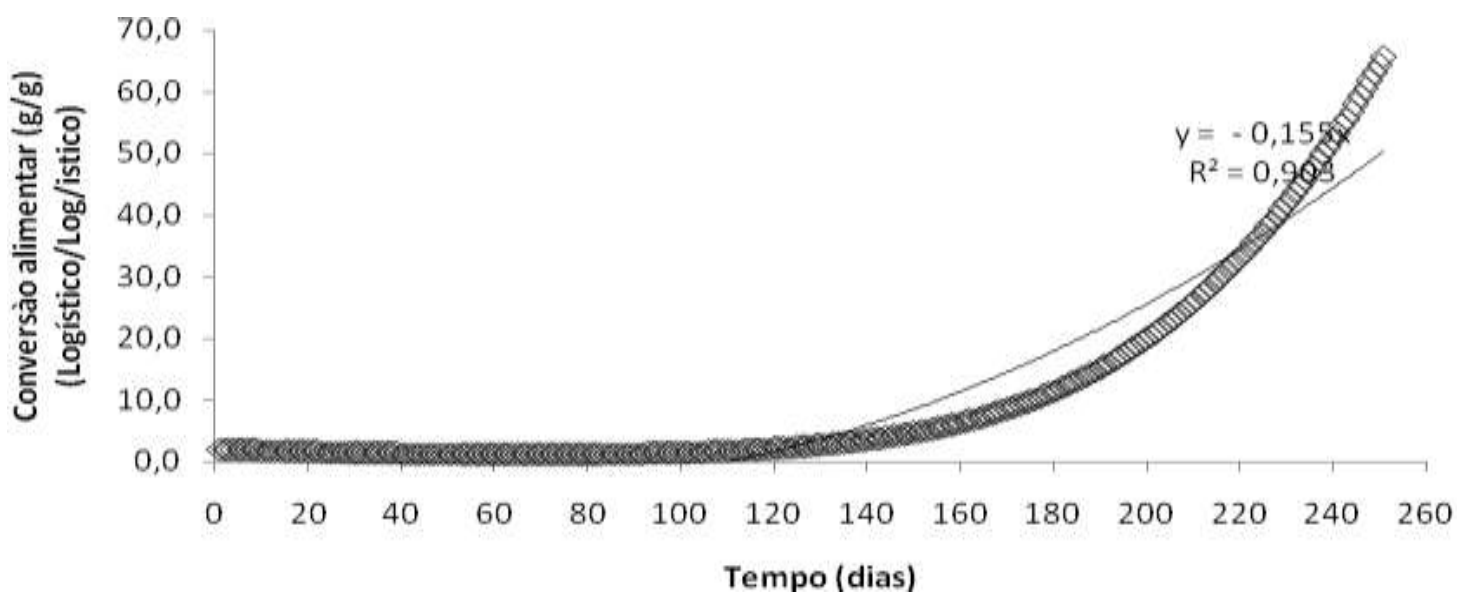

Fonte: Autores.

Figura 9. Estimativa de conversão alimentar para tambaqui (Colossoma macropomum) a partir de valores estimados para crescimento (g) pelo modelo Logístico e dos valores estimados pelo modelo Logístico para oferta de ração até 180 dias.

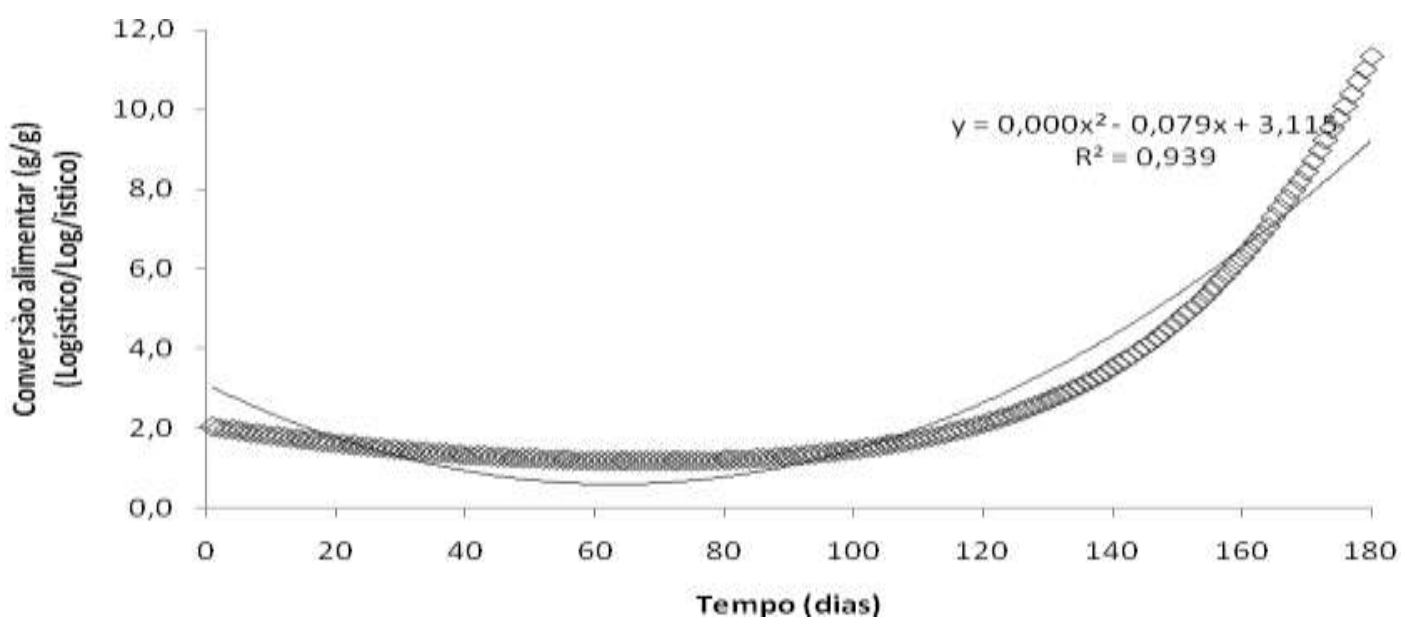

Fonte: Autores.

Houve uma superestimativa dos valores de conversão alimentar na fase final ao adotar o modelo Logístico, apresentando valores fora de realidade de campo no Brasil.

Os valores dos resíduos Studentizados para consumo ou oferta de ração para tambaqui através do modelo Logístico foram adequados dentro das possibilidades e não apresentaram outlier (Figura 10). Os resíduos não devem seguir uma tendência, devem sub ou super estimar os valores de forma que não apareça outleirs ou que somente segue um tendência para cima ou para baixa no eixo X do gráfico (Mansano et al., 2012). 
Figura 10. Valores de resíduos studentizados calculados para estimativas do modelo Logístico para oferta de ração para tambaqui (Colossoma macropomum).

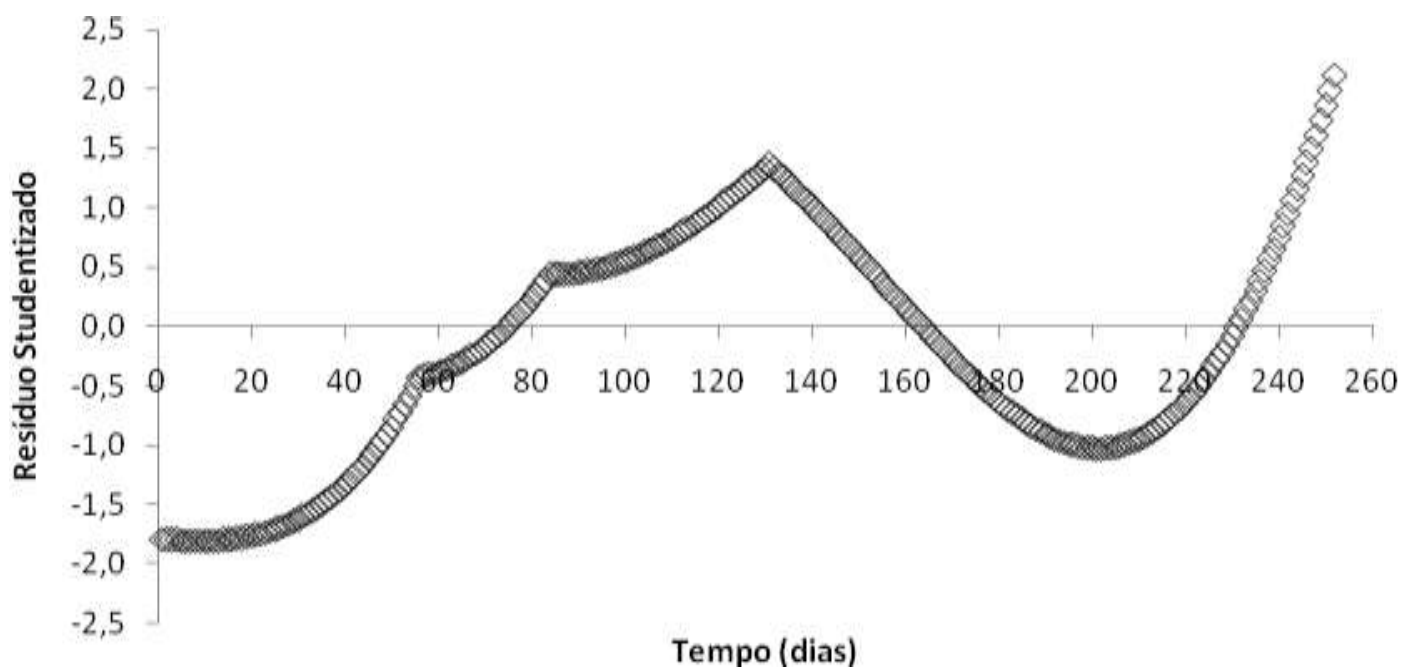

Fonte: Autores.

Importante salientar que os resultados encontrados na literatura, para trabalhos que visaram encontrar equações que representam o crescimento, podem variar dentre as diversas espécies e das condições adotadas (Hota, 1994).

A escolha de um modelo de crescimento adequado é importante, uma vez que pode ter efeito decisivo sobre os resultados de uma simulação de um modelo de dinâmica ecológica. Por exemplo, o modelo Logístico é indicado para descrever o crescimento em períodos curtos de tempo (dias e meses) e em ambientes que possuam algum controle como a nutrição (Gamito, 1998), no caso dos peixes de corte o tempo é considerado curto.

O conjunto adotado de avaliadores de ajustes foi satisfatório para auxiliar na tomada de decisão da escolha do melhor modelo estudado. Critérios de avaliação para seleção de um modelo adequado devem ser bem adotados, pois informações dadas pelos avaliadores de qualidade de ajuste podem indicar qual modelo é o mais apropriado para descrever o crescimento corporal de uma população (Silva et al., 2002; Mendes et al., 2009; Silveira et al., 2011), a interpretação biológica neste trabalho mostrou como é importante essa avaliação.

Em animais não submetidos a restrições alimentares, a relação do peso com a idade ou o tempo resulta numa curva de crescimento com característica sigmoidal, consistindo em três partes diferenciadas: uma fase inicial acelerada, uma fase intermediária linear e uma fase de desaceleração final quando o animal atinge a maturidade. A taxa de crescimento (ganho de peso por unidade de tempo, geralmente em g ou kg dia-1) varia com a idade, aumentando durante a fase acelerada até atingir um máximo na fase intermediária, em que é relativamente constante. Na última fase, a taxa de crescimento diminui progressivamente até zero, quando o animal atinge o corpo maduro ou peso assintótico (López, 2008), esse item final relatado, é que pode levar aos modelos superestimarem valores a serem oferecidos aos animais, e levar a números altíssimos de conversão alimentar ao final do ciclo.

Os modelos não lineares de crescimento e os processos de deposição de nutrientes em peixes têm contribuído para formulação de dietas para os mesmos (Hua et al., 2010), e se forem ajustados para oferecimento da ração aos peixes, aumentarão a eficiência de produção. 


\section{Conclusão}

Modelos matemáticos como a equação de Gompertz e Logístico podem ser utilizados para estimar consumo ou oferta de ração para tilápia do Nilo e tambaqui, porém é necessário rigor nos critérios de avaliação e ajustes.

\section{Referências}

Araujo-Lima, C. A. R. M., \& Goulding, M. Os frutos do tambaqui: ecologia, conservação e cultivo na Amazônia. Tefé, AM: Sociedade Civil Mamirauá, Brasília CNPq-MCT. 1998.186p.

Chizzotti, M. L., \& Valente, E. E. L. (2014) Zootencia de precisão: Uso de sensores na fazenda para maximizar a produtividade. In..XXIV Congresso Brasileiro de Zootecnia, Vitória-ES. Anais... ZOOTEC, 2014

Cyrino, J. E. P., Bicudo, A. J. A., Sado, R. Y., Borghesi, R., \& Dairiki, J. K. A piscicultura e o ambiente: o uso de alimentos ambientalmente corretos em piscicultura. Revista Brasileira de Zootecnia, 39, 68-87, http://dx.doi.org/10.1590/s1516-35982010001300009.

Dumas, A., France, J., \& Bureau, D. Modelling growth and body composition in fish nutrition: where have we been and where are we going? Aquaculture Research. v. 41, p. 161- 181, 2010.

El-Sayed, A. M. Effects of stocking density and feeding levels on growth and feed efficiency of Nile tilapia (Oreochromis niloticus L.) fry. Aquaculture Research, 33(8), 621-626. Wiley. http://dx.doi.org/10.1046/j.1365-2109.2002.00700.x.

Gamito, S. Growth models and their use in ecological modelling: an application to a fish population. Ecological Modelling, 113, 83-94, 1998.

Hartley, H. O. (1961) The modified Gauss Newton method for the fitting of non linear regression functions by least squares. Thechnometrics, $3(2), 269280$.

Hossain, M. A. R., Haylor, G. S., \& Beveridge, M. C. M. (2001). Effect of feeding time and frequency on the growth and feed utilization of African catfish Clarias gariepinus (Burchell 1822) fingerlings. Aquaculture Research, 32(12), 999-1004, Wiley. http://dx.doi.org/10.1046/j.1365-2109.2001.00635.x.

Hota, A. K. (1994). Growth in amphibians. Gerontology, 40(2), 147-160.

Kim, T. N. T. Feeds, water quality, gut morphology and digestion in Nile tilapia (Oreochromis niloticus). Wageningen University. 2017.

Kubitza, F., (2000). Tilápia: tecnologia e planejamento na produção comercial. 285p.

Lazur A. J. (2007). Gold Aquaculture Practices Program: growout pond and water quality management.University of Maryland, College Park, MD, USA.

Lemonte, A. J. (2018). Diagnóstico em regressão normal linear: princípios e aplicação. Revista Brasileira de Biometria, $26,7-26$.

Logato, P. R. P. (2000). Nutrição e Alimentação de Peixes de Água Doce. Aprenda Fácil Editora, 115p.

López, S. (2008). Non-linear functions in animal nutrition. In: France, J., Kebreab, E. Mathematical modelling in animal nutrition. Cambridge: CABI, 47-88.

Mansano, C. F. M., Stéfani, M. V., Pereira, M. M., \& Macente, B. I. Non-linear growth models for bullfrog tadpoles. Ciência e Agrotecnologia 36, 454-462, 2012. https://doi.org/10.1590/S1413-70542012000400010

Mendes, P.N., Muniz, J. A., Silva, F.F., Mazzini, A. R. A., \& Silva, N. A. M. (2009). Análise da curva de crescimento difásica de fêmeas hereford por meio da função não linear de Gompertz. Ciência Animal Brasileira, 10(2), 454-461.

Mourad, N. M. N., Costa, A. C., Freitas, R. T. F., Serafini, M. A., Reis Neto, R. V., Felizardo, V. O. (2018). Weight and morphometric growth of Pacu (Piaractus mesopotamicus), Tambaqui (Colossoma macropumum) and their hybrids from spring to winter. Pesquisa Veterinária Brasileira, 38(3)544-550, http://dx.doi.org/10.1590/1678-5150-pvb-4808.

Pereira, M. M., Mansano, C. F. M., Silva, E. P., \& Stéfani, M. V. Growth in weight and of some tissues in the bullfrog: fitting nonlinear models during the fattening phase. Ciência e Agrotecnologia, 38(6) 598-606. http://dx.doi.org/10.1590/s1413-70542014000600009.

Silva, F. F., Aquino, L. H., \& Oliveira, A. I. G. (2002). Estimativas de parâmetros genéticos de curva de crescimento de gado nelore (Bos indicus). Ciência e Agrotecnologia, Edição Especial, 1562-1567.

Silveira, F. G., Silva, F. F., Carneiro, P.L.S., \& Malhado, C. H. M. (2011). Classificação multivariada de modelos de crescimento para grupos genéticos de Ovinos de corte. Revista Brasileira de Saúde e Produção Animal, 13(1), 62-73.

Sousa, R. M. R., Agostinho, C. A., Oliveira, F. A., Argentim, D., Novelli, P. K.., \& Agostinho, S. M. M. (2012). Productive performance of Nile tilapia (Oreochromis niloticus) fed at different frequencies and periods with automatic dispenser. Arquivo Brasileiro de Medicina Veterinária e Zootecnia, 64(1), 192-197, 2http://dx.doi.org/10.1590/s0102-09352012000100027.

Souza, G. S. (1998). Introdução aos modelos de regressão linear e não-linear. Brasília: Serviço de Produção de Informação/ EMBRAPA-SPI/EMBRAPASEA. 1998. 505 p.

Tsadik, G., \& Kutty, M. N. (1987). Influence of ambient oxygen on feeding and growth of Tilapia, (Oreochromis niloticus) ARAC/87/WP/10.United Nation Development Programme, Food and Agriculture Organization of the United Nations, Nigerian institute for oceanography and marine research project RAF/87/009. 1987. 13p 
Research, Society and Development, v. 10, n. 14, e514101418850, 2021

(CC BY 4.0) | ISSN 2525-3409 | DOI: http://dx.doi.org/10.33448/rsd-v10i14.18850

Val, A. L., Paula-Silva, M. N., \& Almeida-Val, V. M. F. (1998). Hypoxia adaptation in fish of the Amazon: a never-ending task. South African Journal of Zooogy, 33(2)107-114.

Workagegn, K. B. (2019). Evaluation of Growth Performance, Feed Utilization Efficiency and Survival Rate of Juvenile Nile tilapia, Oreochromis niloticus (Linnaeus, 1758) Reared at Different Water Temperature. International Journal of Aquaculture, 2(1), 59-64, Sophia Publishing Group, Inc. http://dx.doi.org/10.5376/ija.2012.02.0009.

Zardin, A. M. S. O., Oliveira C. A. L., Oliveira S. N., Yoshida G. M., Albuquerque D. T., Campos C. M., \& Ribeiro P. R. (2019). Growth curves by Gompertz nonlinear regression model for male and female Nile tilapias from different genetic group. Aquaculture, 511. 734243, https://doi.org/10.1016/j.aquaculture.2019.734243 